\title{
Comparison of alkene hydrogenation in carbon nanoreactors of different diameters: probing the effects of nanoscale confinement on ruthenium nanoparticle catalysis
}

Mehtap Aygun, ${ }^{a}$ Craig T. Stoppiello, ${ }^{a}$ Maria. A. Lebedeva, ${ }^{a, b}$ Emily F. Smith, ${ }^{c}$ Maria del Carmen Gimenez-Lopez, ${ }^{a}$ Andrei N. Khlobystov. ${ }^{\mathrm{a}, \mathrm{c}}$ and Thomas W. Chamberlain ${ }^{\mathrm{d} *}$.

${ }^{a}$ School of Chemistry, University of Nottingham, University Park, Nottingham, UK, NG7 2RD.

${ }^{b}$ Department of Materials, University of Oxford, 16 Parks Road, Oxford, OX1 3PH, UK.

'Nanoscale \& Microscale Research Centre, University of Nottingham, University Park, Nottingham, UK, NG7 2RD.

${ }^{d}$ Institute of Process Research and Development, School of Chemistry, University of Leeds, Leeds, UK, LS2 9JT.

*T.W.Chamberlain@leeds.ac.uk

\begin{abstract}
The catalytic properties of ruthenium nanoparticles (RuNPs) supported in carbon nanoreactors of different diameters - single walled carbon nanotubes (SWNTs, width of cavity $1.5 \mathrm{~nm}$ ) and hollow graphitised nanofibers (GNFs, width of cavity 50-70 nm) - were evaluated using exploratory alkene hydrogenation reactions and compared to RuNPs adsorbed on the surface of SWNT or deposited on carbon black in commercially available $\mathrm{Ru} / \mathrm{C}$. Supercritical $\mathrm{CO}_{2}$ is shown to be essential to enable efficient transport of reactants to the catalytic RuNPs, particularly for the very narrow RuNP@SWNT nanoreactors. Though the RuNPs in SWNT are observed to be highly active, they simultaneously reduce the accessible volume of very narrow SWNTs by 30-40 \% resulting in lower overall turnover numbers (TONs). In contrast, RuNPs confined in wider GNFs were completely accessible and demonstrated remarkable activity compared to unconfined RuNPs on the outer surface of SWNTs or carbon black. Control of the nanoscale environment around the catalytic RuNPs significantly enhances the stability of the catalyst and influences the local concentration of reactant molecules in close proximity to the RuNPs, illustrating the comparable importance of confinement to that of metal loading and size of NPs in the catalyst. Interestingly, extreme spatial confinement also appeared not to be the best strategy for controlling the selectivity of hydrogenations in a competitive reaction of norbornene and benzonorbornadiene, with wider RuNP@GNF nanoreactors displaying enhanced selectivity for the hydrogenation of the aromatic group containing alkene (benzonorbornadiene). This is attributed to the presence of nanoscale graphitic step-edges within the GNF making them an attractive alternative to the extremely narrow SWNT nanoreactors for preparative catalysis.
\end{abstract}


Keywords Nanoreactor, carbon nanotubes, confinement, hydrogenation, heterogeneous catalysis.

\section{Introduction}

Gaining control of chemical reactions in order to improve the yield of a particular product and/or change the reaction pathway is a great challenge in chemical synthesis. One method commonly used to achieve this is to perform reactions in sterically confined environments using materials such as zeolites, porous silica or alumina, molecular cages and carbon nanomaterials to act as nanoscale reaction vessels or nanoreactors. ${ }^{1-9}$ All of these systems have nanosized pores, holes or channels of different geometries and diameters which are capable of accommodating reactants and affecting the distribution of products by imparting some form of steric effect on the transition states or intermediates of reactions.

Carbon nanostructures (CNS) have recently become of great interest for use as nanoreactors in a variety of different catalytic chemical reactions as they are robust, chemically inert, and available in a large range of well-defined pore shapes and sizes. Most importantly, due to recent advances in production, CNS are now readily available in large quantities at low cost which opens up the potential for application in large-scale preparative syntheses for the first time. ${ }^{10-12}$

In addition to acting as reaction vessels and templates to the formation of specific products, CNS also make the ideal support materials for metal nanoparticle (MNP) catalysts. By immobilizing catalytic NPs inside CNS it is possible to combine all of the advantages of nanoreactors, offering control of the size and shape of the reaction volume, with the inherent advantages of heterogeneous catalysis, i.e. enhanced stability and recyclability of metal nanoparticles. ${ }^{13-17}$ As a result a significant number of studies have reported the application of multi-walled carbon nanotubes (MWNTs) and hollow graphitised nanofibers (GNFs), with internal channels ranging from 5-50 $\mathrm{nm}$, as nanoscale reaction vessels and flow reactors for catalytic chemical reactions. In which their commensurate hollow structure facilitates encapsulation of the metal nanoparticles inside the nanotube and provides the perfect environment for reactions to occur within a strictly controlled nanoscale volume. ${ }^{9,17-22}$ Serp et al. performed the selective hydrogenation of cinnamaldehyde to cinnamyl alcohol over a bimetallic Pt-Ru catalyst confined inside MWNTs and compared the metal catalyst activity with both the same sized free standing MNPs and MNPs supported on the outside surface of MWNTs. ${ }^{17} \mathrm{~A}$ significant increase in catalytic performance with higher turnover frequency and selectivity for cinnamyl alcohol was observed for the catalyst inserted in MWNTs as a result of the confinement and enrichment of reactant concentration inside the nanotubes due to stronger interactions between the molecules and the internal surface of the carbon nanostructures. Pan et al. reported that Rh nanoparticles confined inside carbon nanotubes substantially enhanced ethanol conversion compared with the same catalyst located outside of the nanotubes. ${ }^{9}$ Moreover, platinum nanoparticles both inserted into GNFs and adsorbed on to the outside surface were probed in the competitive hydrosilylation reaction of phenylacetylene by Solomonsz et al., demonstrating significant changes in the selectivity of reactions of aromatic and aliphatic molecules within the nanoreactor. ${ }^{18}$ 
The latest studies reveal that the size and shape of the nanotube channel enhances the stability and selectivity of the confined NP catalysts allowing strict control of the nanoparticle size, functionality and reactivity by providing stabilization to the NPs, preventing aggregation into larger particles and bulk metal. ${ }^{23-26}$ Less is known about the use of narrower SWNTs and double-walled carbon nanotubes (DWNTs), with an internal mean diameter of 1-2 nm, however it has been shown that they enable the formation of small, highly stable metal nanoparticles. ${ }^{25}$ The advantageous nature of MNPs@SWNT catalysts, in which the cavity dimensions are commensurate with the size of small organic reactant molecules, is that they impart significant steric influence on reaction pathways thus exhibiting greater effects on the products of reactions than wider MWNTs. We recently carried out hydrogenation reactions of alkenes catalyzed by RuNPs confined within extremely narrow SWNTs and observed very high yields of the products as a result of an enrichment of reactant species inside the nanonoreactor due to confinement effects. ${ }^{27}$ Narrow DWNTs (nanotube diameter of 1-1.5 nm) were utilised to stabilise $\mathrm{Pd}-\mathrm{V}$ bimetallic nanoparticles and the resultant catalyst was shown to be more active for benzene hydroxylation than the same NPs in wider, and hence less confined MWNT (nanotube diameter $=4-8 \mathrm{~nm}$ ). ${ }^{28}$ Similar enhancements in activity as a result of extreme nanotube confinement have also been reported for sub-nanometre titania NPs in DWNTs for propylene epoxidation ${ }^{29}$ and Re NPs in DWNT for benzene hydroxylation. ${ }^{30}$

There are also a few examples of non-catalysed chemical reactions within SWNT, such as a study by Miners et al. who reported the effect of SWNT diameter on the selectivity of $N$-phenylacetamide bromination within SWNT and showed that the inner cavity of the nanotube changed the regioselectivity and activity of a bromination reaction. ${ }^{10,}$ 31-32 These studies demonstrate that the extreme confinement imposed by the unique reaction environment of SWNT-based nanoreactors can significantly alter the selectively and rate of chemical reactions. In addition the SWNT support changes the chemical and physical properties of the NP catalyst, allowing strict control of the NP functionality and reactivity as well as NP size. ${ }^{27}$

Thus, confinement of catalytic processes in very narrow carbon nanoreactors has been shown to control the size and shape selectivity of hydrogenation reactions. In this study we explore the effects of such extreme confinement on the mass transport of reactants and products into and out of SWNT-based catalytic nanoreactors and compare this to GNFs, which are wider, and therefore more accessible to reactants and crucially, in contrast to MWNTS, have structured internal channels which can also influence chemical reactions.

\section{Experimental}

SWNTs, GNFs and C60 were purchased from Helix Material Solutions (Arc-discharge, USA), Pyrograf Products Inc (PR19, chemical vapor deposition, USA) and SES Research (USA), respectively. All other reagents and solvents were purchased from Sigma-Aldrich (UK) and used without further purification. All of the glassware required to perform the experiments was thoroughly cleaned with 'aqua regia' (concentrated hydrochloric and nitric acids (3:1)) and rinsed with deionized water prior to use.

\section{Catalyst preparation:}


SWNTs were annealed at $380^{\circ} \mathrm{C}$ for 20 minutes to open their termini and remove any residual amorphous carbon from the internal cavities; a 20-30\% weight loss was observed prior to use. Average SWNT length after thermal treatment was reported previously to be $2.43 \pm 0.85 \mu \mathrm{m} .{ }^{33} \mathrm{GNFs}$ were thermally annealed at $450{ }^{\circ} \mathrm{C}$ for 1 hour prior to use. The average length after thermal treatment was measured by TEM to be $15.34 \pm 12.10 \mu \mathrm{m}$, (see Electronic Supplementary Information file (ESI) Figure S-2).

RuNPs@SWNT and RuNPs@GNF - The metal carbonyl precursor, Ru3 $(C O)_{12}(1.05$ mg/0.21 mg, masses equivalent to the wt.\% of Ru metal required for SWNT and GNF respectively) were combined with freshly opened SWNTs $(10 \mathrm{mg})$ or GNFs $(10 \mathrm{mg})$ in a quartz ampoule, and sealed under vacuum $\left(10^{-6} \mathrm{bar}\right)$ heated at $140{ }^{\circ} \mathrm{C}$ for 3 days. After 3 days, the sample inside the quartz ampoule was cooled by immersing in an ice bath. The sample was then removed from the ampoule, sonicated in tetrahydrofuran $(10 \mathrm{~mL})$ for 15 minutes, then filtered through a PTFE membrane filter (pore size $0.2 \mu \mathrm{m}$ ) and repetitively washed with tetrahydrofuran $(3 \times 10 \mathrm{~mL}$ ). After washing, the sample was sealed in a quartz ampoule under an argon atmosphere and heated at $600{ }^{\circ} \mathrm{C}$ for 2 hours to decompose the metal carbonyl into the desired pure metal nanoparticles.

RuNPs/(C60@SWNT) - Freshly opened SWNT (10 mg) and C $60_{6}(20 \mathrm{mg})$ were sealed under vacuum $\left(10^{-6} \mathrm{bar}\right)$ in a quartz ampoule and heated at $500{ }^{\circ} \mathrm{C}$ for 2 days. After 2 days, the sample was removed from the ampoule and sonicated in toluene $(10 \mathrm{~mL}$ ) for 15 minutes. The sample was then filtered through a nylon membrane filter (pore size $0.2 \mu \mathrm{m})$ and repetitively washed with toluene $(3 \times 10 \mathrm{~mL})$. After washing, the $\mathrm{C}_{60} @ S W N T(10 \mathrm{mg})$ and $\mathrm{Ru}_{3}(\mathrm{CO})_{12}(0.65 \mathrm{mg}$, a mass equivalent to the wt.\% of $\mathrm{Ru}$ metal required) were combined in a quartz ampoule and sealed under an argon atmosphere and heated at $600{ }^{\circ} \mathrm{C}$ for 2 hours decompose the metal carbonyl into the desired pure metal nanoparticles.

HRTEM analysis was performed on a JEOL 2100 Field emission gun microscope with an information limit of 0.12 $\mathrm{nm}$ at $100 \mathrm{kV}$ or $200 \mathrm{kV}$. High resolution scanning transmission microcpy (HRSTEM) images were acquired using the JEOL digital STEM system. Samples (RuNPs@SWNT, RuNPs/C60@SWNT, RuNPs@GNF and Ru/C) were prepared for TEM analysis by dispersing the materials in HPLC grade iso-propanol using ultra-sonication, then drop casting the resultant suspension onto a lacey carbon film coated copper grid. ${ }^{1} \mathrm{H}$ NMR spectra were recorded using a Bruker DPX300 NMR spectrometer. ${ }^{1} \mathrm{H}$ NMR spectra were taken in $\mathrm{CDCl}_{3}$ and were referenced to residual trimethysilane $(\mathrm{TMS})(0 \mathrm{ppm})$ and reported as follows: chemical shift, multiplicity $(\mathrm{s}=$ singlet, $\mathrm{d}=$ doublet, $\mathrm{t}=$ triplet, $\mathrm{dd}=$ doublet of doublet, $\mathrm{m}$ = multiplet). TGA analysis was performed on a TA Instruments TGA-SDTQ600 analyser. Samples for TGA analyses were heated in an inert atmosphere up to $1000{ }^{\circ} \mathrm{C}$ with a heating rate of $10^{\circ} \mathrm{C} / \mathrm{min}$. The powder X-ray diffraction (XRD) patterns were obtained using a PANanalytical $X^{\prime}$ Pert PRO diffrctometer equipped witha Cu-Ka radiation source operating at $40 \mathrm{kV}$ and $40 \mathrm{~mA}$, with $0.05252^{\circ}$ step size and 5925.18 second step time. Surface area analysis was performed using the Brunauer-Emmett-Teller (BET) method based on adsorption data in the relative pressure (P/Po) range 0.02 to 0.22 by measuring nitrogen sorption isotherms of the samples $(50 \mathrm{mg})$ at $-196{ }^{\circ} \mathrm{C}$ on a Micromeritics ASAP 2020 sorptometer. X-ray photoelectron spectrocopy (XPS) samples were analysed using the Kratos AXIS ULTRA with a mono-chromated Al k $\alpha$ X-ray source (1486.6eV) operated at $10 \mathrm{~mA}$ emission current and $12 \mathrm{kV}$ anode potential (120 W). 


\section{Benzonorbornadiene (2a) synthesis by Diels Alder reaction}

Benzonorbornadiene was synthesized according to the previously reported procedure. ${ }^{34} 1,2$-Dibromobenzene $(5.00 \mathrm{~g}, 21.45 \mathrm{mmol})$ and cyclopentadiene $(1.42 \mathrm{~g}, 21.45 \mathrm{mmol})$ were stirred in toluene $(13 \mathrm{~mL})$ at $0{ }^{\circ} \mathrm{C}$ under $\mathrm{Ar}$. $n$-BuLi (12 mL, 1.78M in hexanes, $21.45 \mathrm{mmol}$ ) was added to this solution dropwise over 30 min during which the reaction solution became first yellow then cloudy white. After an additional $10 \mathrm{~min}$ at $0{ }^{\circ} \mathrm{C}$ the mixture was allowed to warm to room temperature, stirred overnight and treated with $\mathrm{H}_{2} \mathrm{O}(20 \mathrm{~mL})$ and extracted with hexanes $(3 \times 30 \mathrm{~mL})$. The organic layer was dried over $\mathrm{MgSO}_{4}$, filtered, and concentrated to obtain yellow oil. The product was purified by chromatography on silica gel eluting with hexanes to provide a clear and colourless oil (1.6 g, $0.11 \mathrm{mmol}, 65 \%)$.

Benzonorbornadiene (1,4-dihydro-1,4-methano-naphthalene) (2a); ${ }^{1} \mathrm{H} \mathrm{NMR}\left(300 \mathrm{MHz}, 297 \mathrm{~K}, \mathrm{CDCl}_{3}, \delta, \mathrm{ppm}\right)$ : 7.24-7.21 (m, $2 \mathrm{H}), 6.95-6.92(\mathrm{~m}, 2 \mathrm{H}), 6.80(\mathrm{~m}, 2 \mathrm{H}), 3.90(\mathrm{~m}, 2 \mathrm{H}), 2.34-2.30(\mathrm{~m}, 1 \mathrm{H}), 2.26-2.23(\mathrm{~m}, 1 \mathrm{H})$.

\section{Hydrogenation reactions using lab glassware:}

All reactions were performed in a pyrex pressure tube (10 bar) with a stirring bar. RuNPs@carbon nanoreactor (in each case an amount equivalent $0.0017 \mathrm{mmol} \%$ of Ru in the reaction mixture) was suspended in cyclooctane $(1 \mathrm{~mL})$ in the bottom of the tube and the resulting mixture was saturated with $\mathrm{H}_{2}$ by bubbling a mixture of $10 \%$ $\mathrm{H}_{2}$ and $90 \%$ Argon gas ( 1 bar) through the solution at $25^{\circ} \mathrm{C}$ for 30 minutes. The alkene $(0.05 \mathrm{~mL})$ was then added to the resulting $\mathrm{H}_{2}$ saturated solution. The tube was sealed and the resultant suspension was heated at $110{ }^{\circ} \mathrm{C}$ for 24 hour. After this, the reaction was stopped and the mixture was cooled down to room temperature. The resultant material was analyzed by ${ }^{1} \mathrm{H}$ NMR spectroscopy.

\section{Hydrogenation reactions using a high pressure $\mathrm{scCO}_{2}$ batch reactor:}

In general, RuNPs@carbon nanoreactor (0.0017 mmol\% of Ru) and alkene substrate (1 mmol) were put into a high pressure reactor $(10 \mathrm{~mL})$. The reactor volume was degassed thoroughly with $\mathrm{H}_{2}$ for 30 min. Then, the reactor was sealed and pressurized with $\mathrm{H}_{2}(10 \mathrm{bar})$ and heated to $110{ }^{\circ} \mathrm{C}$ by adding $\mathrm{CO}_{2}(100 \mathrm{bar})$ and left for 24 hour. At the end of the reaction, the reactor was cooled and depressurized. The resultant material was analyzed by ${ }^{1} \mathrm{H}$ NMR spectroscopy. All catalytic reactions were performed in duplicate, and the yields given are averages of the two experiments.

Norbornane (1b); ${ }^{1} \mathrm{H}$ NMR (300 MHz, $\left.297 \mathrm{~K}, \mathrm{CDCl}_{3}, \delta, \mathrm{ppm}\right): 2.21(\mathrm{~m}, 2 \mathrm{H}), 1.50(\mathrm{~m}, 4 \mathrm{H}), 1.18(\mathrm{~m}, 6 \mathrm{H})$.

1,4-Methano-1,2,3,4-tetrahydronaphthalene (2b); ${ }^{1} \mathrm{H}$ NMR (300 MHz, $\left.297 \mathrm{~K}, \mathrm{CDCl}_{3}, \delta, \mathrm{ppm}\right):$ 7.30-7.10 (m, $4 \mathrm{H}$, ArH), 3.50-3.35 (m, 2H, CH), 2.10-1.50 and 1.50-1.10 (m, 6H, $\left.\mathrm{CH}_{2}\right)$.

\section{The use of high pressure $\mathrm{scCO}_{2}$ batch reactor:}

In general, the autoclave reactor was loaded with RuNPs@SWNT and norbornene substrate (1 mmol) and an O-ring was placed to seal the reactor. The reactor volume was degassed thoroughly with $\mathrm{H}_{2}$ for 30 min by opening 
the outlet valve. Then, the reactor was sealed and pressurized with $\mathrm{H}_{2}$ (10 bar) and heated initially to $40{ }^{\circ} \mathrm{C}$ in order to add the $\mathrm{CO}_{2}\left(50 \mathrm{bar}\right.$ ) as a supercritical phase using a pickle pump and then heated slowly to $110{ }^{\circ} \mathrm{C}$ to make sure that overall pressure is 100 bar by adding more $\mathrm{scCO}_{2}$ at this temperature. The reaction was then left for 24 hour and cooled under room temperature to depressurize slowly by opening outlet valve.

\section{Result and Discussion}

In our study, a simple and efficient approach for catalyst synthesis in the inner cavity of carbon nanotubes is used to create very active Ru nanoparticles within an extremely constrained carbon nanoreactor environment. ${ }^{35}$ In this approach, $\mathrm{Ru}_{3}(\mathrm{CO})_{12}$ molecules inserted from the vapour phase into the freshly opened SWNT are decomposed to form Ru nanoparticles within the nanotube (RuNPs@SWNT). High resolution transmission electron microscopy (HRTEM) imaging of the resultant hybrid nanomaterial RuNPs@SWNT reveals the shape, size and location of Ru nanoparticles inside the nanoreactor. HRTEM confirmed that the RuNPs were located mostly inside the SWNT, where the nanotube sidewall stabilises and templates the formation of NPs resulting in small, well defined particles with a narrow size distribution, $\left(d_{N P}=0.74 \pm 0.18 \mathrm{~nm}\right.$, Table 1$)$. A combination of thermogravimetric analysis (TGA) and inductively coupled plasma optical emission spectrometry (ICP-OES) was used to determine the precise loading of Ru in RuNPs@SWNT showing the material to be $1.6 \%$ Ru by weight. This represents the maxiumum loading of Ru nanoparticles into SWNT with the remaining Ru material deposited and subsequently washed from the outer walls of the nanoutbes prior to nanoparticle formation, an essential step to ensure no unconfined nanoparticles are present to take part in the reaction (see supporting information file Figures S-13 and Table S-1). However, TEM reveals a small portion (<10\%) of NPs are located on the outside of the nanotubes where they do not benefit from the same templating effect and therefore are larger and less uniform ( $d_{N P}=2.49 \pm 0.85 \mathrm{~nm}$ (Figure 1a and Figure S-1 in Electronic Supplementary Material). Energy dispersive X-ray spectroscopy (EDX), XPS, and lattice spacing analysis of HRTEM images and powder XRD are consistent with a hexagonally close packed structure of metallic the RuNPs (see Figure 1 and ESI for full details). ${ }^{36}$ 
a)

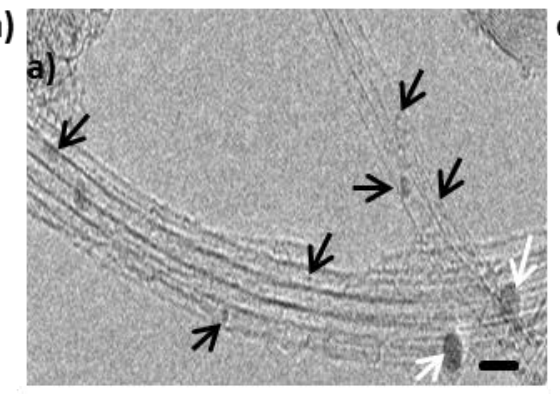

b)

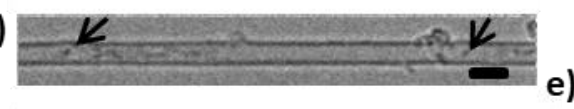

d)

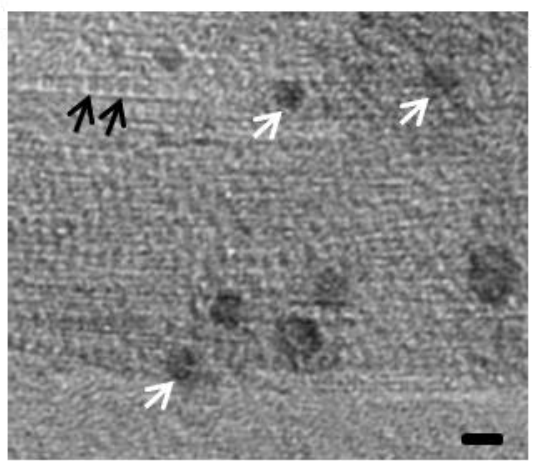

f)

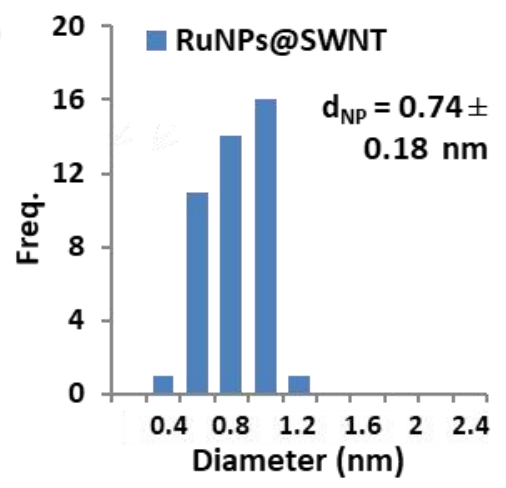

c)
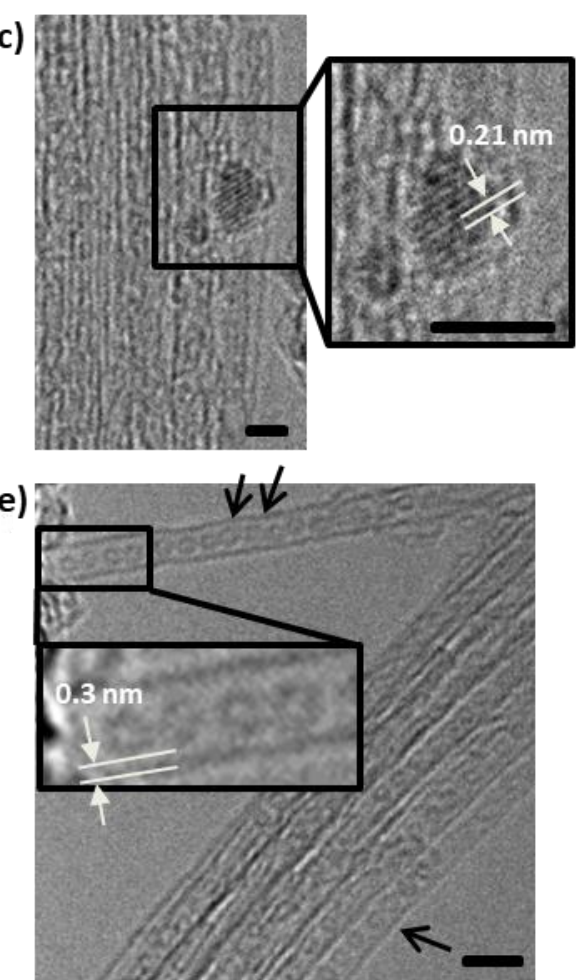

g) 20

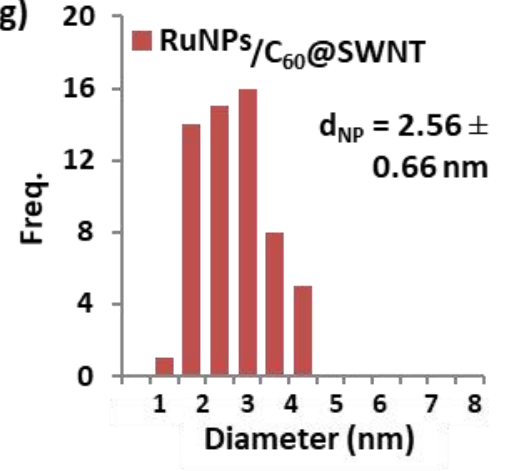

Figure 1. a-c) HRTEM images of RuNPs@SWNT loaded with $1.6 \%$ of Ru by wt. Black and white arrows indicate RuNPs inside and outside of the SWNT respectively, and enlarged region (c) showing the Ru [002] lattice spacing $(\mathrm{d}=0.21 \mathrm{~nm})$ of an individual, hcp structured, metallic NP which corresponds to the $2 \theta$ peak centred at $42.2^{\circ}$ in the XRD (see ESI). d) HRTEM image of RuNPs/C60@SWNT loaded with 3.1 \% of Ru by wt. after filling the SWNT with $\mathrm{C}_{60}$, and e), HRTEM image of the $\mathrm{C}_{60} @ S W N T$ support material showing the internal channels of the SWNT are completely full of $\mathrm{C}_{60}$. Enlarged region (inset) shows only single $\mathrm{C}_{60} @ S W N T$ structure in which a $0.3 \mathrm{~nm}$ van der Waals gap can be observed between $\mathrm{C}_{60}$ molecules and the SWNT wall confirming that there is no space for reactants or Ru NP precursors to fit. White arrows show RuNPs located on the outside of the SWNT and the black arrows show $\mathrm{C}_{60}$ molecules. f) The size distribution of RuNPs located inside the SWNT highlights the narrow diameter distribution of the RuNPs (blue), and $\mathbf{g}$ ), the size distribution of RuNPs on the outside of the SWNT 
(red) is observed to be greater than the confined NPs inside and comparable to the NPs located on the outside of the SWNT in RuNPs@SWNT. Scale bars: 2 nm in all images.

Table 1. Nanoparticle sizing data and theoretical active Ru surface area for all carbon nanoreactor supported RuNP catalysts.

\begin{tabular}{lll}
\hline Catalyst & $\begin{array}{l}\text { Average size of } \\
\text { RuNPs }(\mathrm{nm})\end{array}$ & $\begin{array}{l}\text { Theoretical active Ru } \\
\text { surface area }\left(\mathrm{m}^{2} / \mathrm{g} \text { of }\right. \\
\text { catalyst) }\end{array}$ \\
\hline RuNPs@SWNT & $0.74 \pm 0.18$ & 7.46 \\
RuNPs/C60@SWNT & $2.56 \pm 0.62$ & 4.31 \\
RuNPs@GNF & $3.58 \pm 1.14$ & 1.00 \\
Ru/Cc & $6.63 \pm 2.45$ & 2.69
\end{tabular}

a The active Ru surface area of the RuNPs catalysts was calculated assuming all of the NPs to be the average diameter measured by TEM (see SI file for full details).

The RuNPs@SWNT catalyst was then investigated using exploratory alkene hydrogenation reactions and as the RuNPs are located predominantly within the nanotube channel all reactions are assumed to be performed under the effects of extreme confinement. When selecting an appropriate reactant it is important that the van der Waals size of the reactant molecules is smaller than the $1.5 \mathrm{~nm}$ diameter of the SWNT channel. As the hydrogenation of cycloalkenes and butanal in continuous flow reactors has recently been demonstrated in nanotubes, in this study we selected a bicyclic alkene norbornene (1a) and a tricyclic alkene benzonorbornadiene (2a), both of which have non-planar angular shapes, and in the case of $\mathbf{2 a}$ an additional aromatic ring increasing the steric bulk as compared to 1a (Scheme 1). ${ }^{27}$

The RuNPs@SWNT catalyst was initially tested in the hydrogenation reaction of norbornene using molecular hydrogen in a conventional organic solvent, cyclooctane, at atmospheric pressure using laboratory glassware. Very low catalytic activity was observed for RuNP@SWNT under these conditions (a $10 \%$ yield of norbornane in $24 \mathrm{~h}$ ), and is attributed to the restricted space in the narrow nanotube channels hindering access of the solvent/reactants to the confined metal catalyst. Typically, the use of supercritical $\mathrm{CO}_{2}\left(\mathrm{scCO}_{2}\right) \mathrm{can}$ eliminate such mass transfer problems in nano- or microporous structures, ${ }^{27}$ therefore, a high pressure $\mathrm{scCO}_{2}$ batch reactor system was utilized in which the excellent diffusivity and mass transfer properties of $\mathrm{scCO}_{2} \mathrm{are}$ exploited to efficiently deliver the reagents to the RuNP catalyst surface within the narrow nanoreactors (Figure 2). 


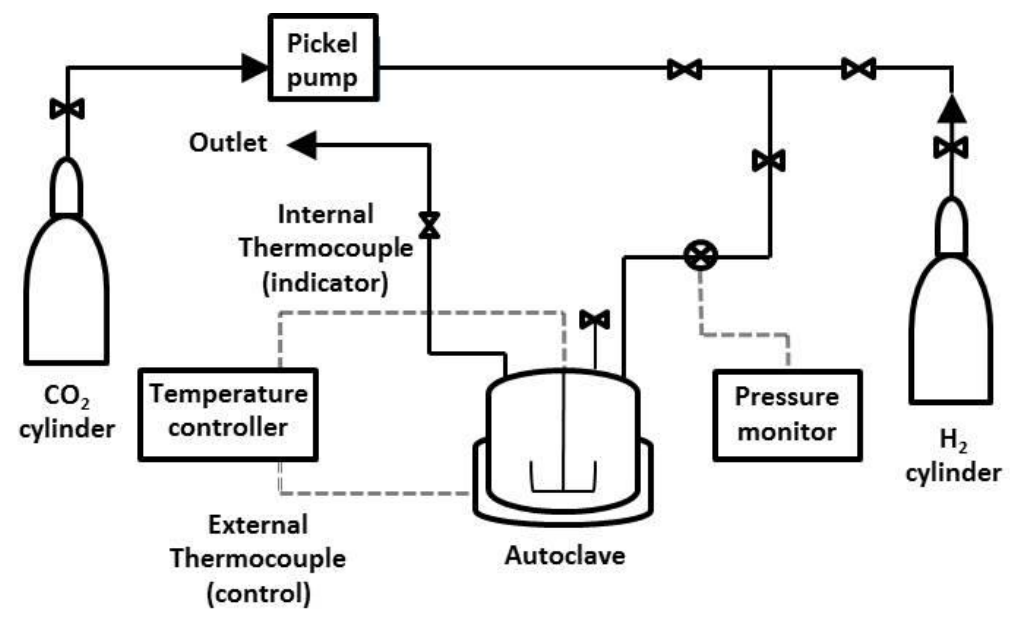

Figure 2. A schematic diagram of the $\mathrm{scCO}_{2}$ hydrogenation batch reactor.

Test hydrogenation reactions were performed using the $\mathrm{scCO}_{2}$ batch reactor in the presence of RuNPs@SWNT, and norbornene showed a higher TON (total number of product molecules formed per available Ru active site, see supporting information for details of how this was calculated), ${ }^{27}$ than the larger benzonorbornadiene under identical reaction conditions (Scheme 1 and Table 2). Control reactions using as-received SWNTs showed no reactivity despite EDX and TEM (see supporting information Figure S-1) showing the presence of residual $\mathrm{Ni} / \mathrm{Y}$ catalyst from nanotube synthesis implying all of the nickel is completely passivated by layers of graphitic carbon shells (Table 2).

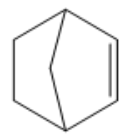

$1 \mathrm{a}$

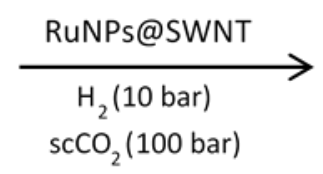

$110^{\circ} \mathrm{C}, 24$ hour

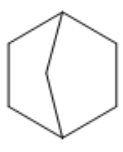

$1 \mathbf{b}$<smiles>C1=CC2CC1c1ccccc12</smiles>

$2 a$

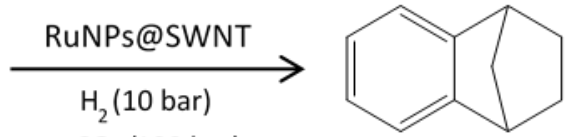

$2 \mathbf{b}$

Scheme 1. Hydrogenation of norbornene and benzonorbornadiene in the presence of RuNPs@SWNT.

Table 2. Hydrogenation reactions of norbornene and benzonorbornadiene in the presence of RuNPs@SWNT, RuNPs/C60@SWNT, RuNPs@GNF and commercial Ru/C using a high pressure $\mathrm{scCO}_{2}$ batch reactor.

\begin{tabular}{|c|c|c|c|}
\hline \multirow{2}{*}{ Catalyst } & \multicolumn{2}{|c|}{ Yield $^{c}$ of Products (\%) / TON } & \multirow{2}{*}{$\begin{array}{l}\text { Ratio of TONs for } \\
\mathbf{1 b} \mathbf{2} \mathbf{b}\end{array}$} \\
\hline & $1 b$ & $2 b$ & \\
\hline $\mathrm{SWNT}^{\mathrm{a}}$ & 0 & N/A & $\mathrm{N} / \mathrm{A}$ \\
\hline RuNPs@SWNT & $91 / 2959$ & $61 / 1983$ & $1.5: 1$ \\
\hline
\end{tabular}




$\begin{array}{llll}\text { C60@SWNT } & 0 & \text { N/A } & \text { N/A } \\ \text { RuNPs/C60@SWNT } & 12 / 675 & 7 / 393 & 1.7: 1 \\ \text { GNF } & 0 & \text { N/A } & \text { N/A } \\ \text { RuNPs@GNF } & 46 / 11216 & 17 / 4145 & 2.7: 1 \\ \text { Ru/C } & 51 / 7428 & 21 / 3058 & 2.4: 1\end{array}$

Reaction conditions: alkene $(1 \mathrm{mmol})$, catalyst (equivalent of $0.0017 \mathrm{mmol}$ of ruthenium in the reaction mixture), $\mathrm{H}_{2}(10$ bar), $\mathrm{scCO}_{2}$ (100 bar), $24 \mathrm{~h}, 110^{\circ} \mathrm{C}$. a SWNT was annealed at $380^{\circ} \mathrm{C}$ for 20 minutes to open their termini prior to use. ${ }^{\mathrm{b}} \mathrm{GNF}$ was annealed at $450{ }^{\circ} \mathrm{C}$ for 1 hour prior to use. ${ }^{\mathrm{C}}$ Yield determined by ${ }^{1} \mathrm{H}$ NMR with an error of $\pm 2 \%$. ${ }^{\mathrm{d}}$ The turnover number (TON) was calculated as the ratio of the number of molecules of substrate consumed in the reaction per number of available Ru active sites in catalyst used in the reaction (see SI file for details of the calculation of the theoretical number of active Ru sites in each catalyst).

Crucially, to verify the precise location of the reaction it was important to investigate the effect of the small portion of larger RuNPs located on the outside of the SWNT which were more readily accessible than the RuNPS confined within the nanotube channel. A control material was therefore synthesised, in which RuNPs are located solely on the outside of SWNT, RuNPs/C60@SWNT. This material was prepared by filling of the internal cavities of the SWNT support material with $\mathrm{C}_{60}$ to block the channels prior to exposure to the RuNP precursor, $\mathrm{Ru}_{3} \mathrm{CO}_{12}$ vapour (see ESI Figure S-4a for HRTEM of the C60 $_{60}$ SWNT support material). HRTEM and gas absorption measurements (Figure S-14-15), which reveal an absence of 1-2 nm pores in the C60@SWNT support, agree with the fact that the $\mathrm{C}_{60}$ molecules completely block the inner channels of the SWNTs, such that the $\mathrm{Ru}_{3} \mathrm{CO}_{12} \mathrm{Can}$ only deposit on the outside of the nanotubes and subsequent thermal treatment causes decomposition of the precursor resulting in RuNPs formation exclusively on the outer surface of the SWNT (Figures 1d and S-4b). Removal of the templating effect of the inner nanotube cavity during NP formation resulted in the Ru nanoparticles of diameter $2.56 \pm 0.66 \mathrm{~nm}$ on the nanotube surface exhibiting a wider size distribution significantly larger as compared to nanoparticles formed inside, but comparible to the NPs located on the outside of, nanaotubes in RuNPs@SWNT (c.f. average NP sizes of $0.74 \pm 0.18 \mathrm{~nm}$ and $2.49 \pm 0.85 \mathrm{~nm}$ for NPs inside and outside respectively). This allowed comparison of the catalytic activity of RuNPs located on the outside of the SWNT (RuNPs/C60@SWNT) with RuNPs located inside the nanotube (RuNPs@SWNT). For reference $\mathrm{C}_{60} @ S W N T$ exhibited no catalytic activity when tested in $\mathrm{ScCO}_{2}$ hydrogenation reactions, (Table 2).

In comparison with RuNPs@SWNT, RuNPs/C60@SWNT showed very low catalytic performance in $\mathrm{scCO}_{2}$ reactions implying that the larger NPs on the outer walls of the nanoreactor are less active than the smaller RuNPs confined within the nanoreactor. ${ }^{37-38}$ Interestingly, the RuNPs on the outside of the C60@SWNT support $\left(\mathrm{d}_{\mathrm{NP}}=2.56 \mathrm{~nm}\right)$ are similar in size to the RuNPs inside the GNF in the RuNPs@GNFs catalyst $\left(\mathrm{d}_{\mathrm{NP}}=3.58 \mathrm{~nm}\right)$, see below for details, but give siginificantly lower reaction yields, (Table 2). It is hypothesised, therefore, that the lack of activity of the RuNPs/C60@SWNT catalyst is most likely a result of the lack of confinement for the reactions, which cannot create a similarly high local concentration of the reactant molecules around the catalyst nanoparticles in RuNPs/C60@SWNT as in RuNPs@SWNT (and RuNPs@GNF) leading to a lower yield of the 
products in the case of the former material. This is consistent with the previously reported examples of enhanced local concentration of reactants inside nanotubes resulting in higher yields and is consistent with our observation of low norbornene conversion ( $10 \%$ ) in the reaction catalyzed by RuNPs@SWNT performed in cyclooctane solvent in which the reaction only takes place on the accessible Ru nanoparticles located on the outside surface of SWNT in the absence of high pressure scCO 2 (c.f. RuNPs/ $\mathrm{C}_{60} @$ SWNT showed 6\% norbornene conversion to norbornane using the same conditions). ${ }^{18-19,26,31-32}$ It is therefore concluded that the RuNPs located inside the carbon nanoreactor are significantly more active and are responsible for the majority of product formation, and thus, the contribution of the minority of RuNPs on the outside of the SWNT ( $10 \%$ by HRTEM) and GNF ( $\sim \%$ by STEM, Figure 3c) for RuNPs@SWNT and RuNPS@GNF catalysts respectively, is neglibible (see ESI for details).

The general observed trend of higher TONs for norbornene, compared to benzonorbornadiene, is most likely due to the steric bulk of the additional benzyl group hindering adsorption of benzonorbornadiene on the Ru NP surface, as well as potentially an electron withdrawing effect of the additional benzene ring compared to norbornene..$^{39}$ Interestingly, however, there is a significant difference in the relative TONs of reactions of $\mathbf{1 b}$ and $\mathbf{2 b}$ for each of the SWNT catalysts, highlighted by considering the ratio of TONs, Table 2, implying the shape of the catalyst affects the reactions in an appreciable way. The extremely confined RuNPs@SWNT system, where the ratio of TONs of $\mathbf{1 b}: \mathbf{2} \mathbf{b}$ is dramatically lower than for the unconfined RuNPs $/ \mathrm{C}_{60} @ S W N T$ system, appears to have a higher affinity for $\mathbf{2 b}$, potentially due to favourable $\pi-\pi$ interactions between the aromatic group and the nanotube sidewall. Cleary understanding the nature and extent of the confinement in these systems is crucial to selecting the correct environment for a specific reaction.

To fully understand the impact of the restricted reaction space within the SWNT, ${ }^{27}$ hydrogenation of norbornene and benzonorbornadiene was performed with catalysts which provide lower (RuNPs@GNF) and no confinement (RuNPs/carbon black) of reactants around the catalytic nanoparticles. To achieve this, GNFs were used, which have an inner diameter of $52 \pm 13 \mathrm{~nm}$ and an outer diameter of $99 \pm 25 \mathrm{~nm}$, and are, like MWNTs, significantly wider than SWNTs, allowing reactants in (and products out) more readily and thus can be considered as providing a lower level of confinement. In addition, unlike the entirely smooth MWNTs, GNFs have a unique step-edge internal structure, which stabilses the RuNPs and has the capacity to impart additional confinement effects on reactions which will be probed in this study. RuNPs within the GNF (RuNPs@GNF) were formed using similar conditions to RuNPs@SWNT, however due to the significantly lower surface area of the GNFs only 1\% Ru by weight was used, to ensure that RuNPs were only formed in the cavity of the GNFs. ${ }^{18}$ In identical fashion to SWNT, it is the internal structure of the GNF which templates the formation of the RuNPs, stabilising the NPs and controlling their size and shape. HRTEM imaging confirms that RUNPs are located solely inside the GNF, at the step-edges formed by rolled-up graphitic sheets, with a mean nanoparticle diameter of $3.58 \pm 1.14 \mathrm{~nm}$ (Figure 3a, Table 1). HRTEM and XRD analyses (see ESI for full details) confirm the metallic nature of the RuNPs and STEM, (Figures $3 \mathrm{c}$ and S-3), approximates the amount of RuNP material on the outer surface to be $7 \%$. XPS reveals a characteristic Ru 3d 5/2 peak at $280.2 \mathrm{eV}$ for all three carbon nanostructure supported RuNPs materials which is consistent with the previously reported literature for metallic ruthenium. ${ }^{40}$ 
In addition, commercially available $\mathrm{Ru} / \mathrm{C}$, containing $5 \% \mathrm{Ru}$ by weight, was used as a control material in which the RuNPs are located on the surface of carbon black which imparts no confinement effects. HRTEM confirmed that the metal nanoparticles are distributed throughout the carbon support in $\mathrm{Ru} / \mathrm{C}$ and showed a wide distribution of NP sizes, with a mean diameter of $6.63 \pm 2.45 \mathrm{~nm}$ (Figure 3f, Table 1). It is important to note that as the RuNPs are not identical sizes across the catalysts in this study, it is not possible to unambiguously assign all differences in reactivity soley to the effect of confinement. However, as the shape and size of the NPs in each material is a direct result of the confinement experienced during formation, and the differences in reactivity cannot be explained solely by considering the size of the NPs, vital information can be obtained by comparing the reactivity of the catalysts.

In similar fashion to the SWNT nanoreactors, the catalytic activities of RuNPs@GNF and Ru/C, along with the as-supplied GNFs as a control, were investigated in hydrogenation of norbornene and benznorbornodiene (Table 2). The empty GNFs displayed no catalytic activity at all. Interestingly, the RuNPs@GNF catalyst exhibited turnover numbers for norbornene (11216) and benzonorbornadiene (4145) conversion, which are significantly higher when compared to RuNPs@SWNT (c.f. (2959) and (1983) respectively), and the unconfined Ru nanoparticles in commercial Ru/C or in RuNPs/C60@SWNT. This is possibly due to the RuNPs inside of the much wider GNF cavity being more accessible for the reactant molecules coupled with the formation of favourable $\pi-\pi$ interactions with both $1 \mathbf{a}$ and $\mathbf{2 a}$ at the step-edge enhancing the local concentration of the reactants near the RuNPs, and therefore the rate of reaction. ${ }^{41}$ 
a)

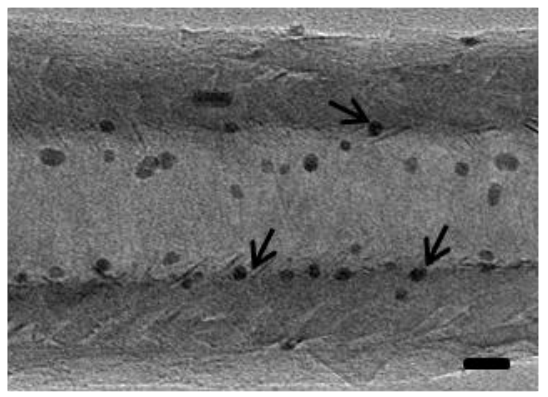

c)

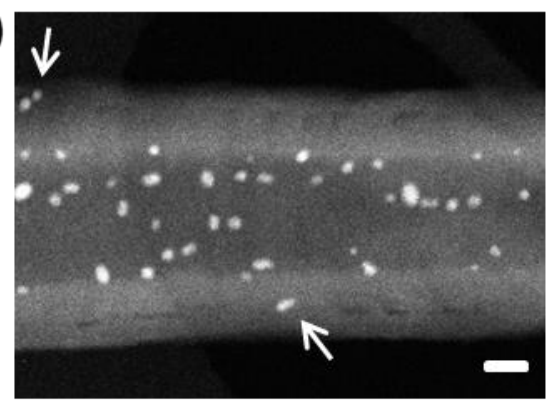

e)

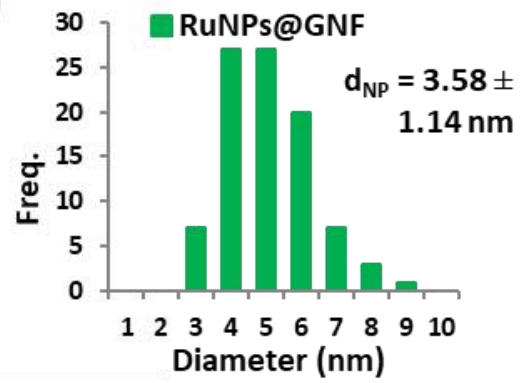

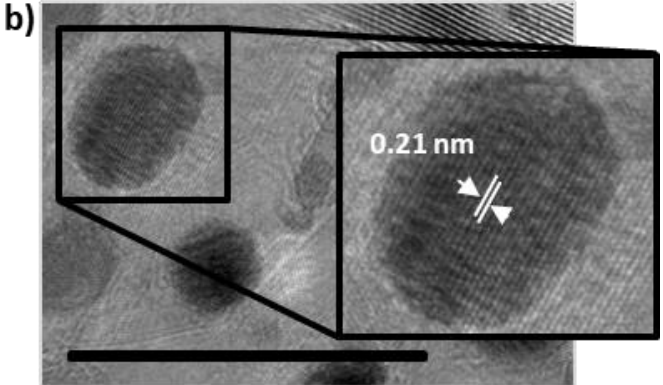

d)
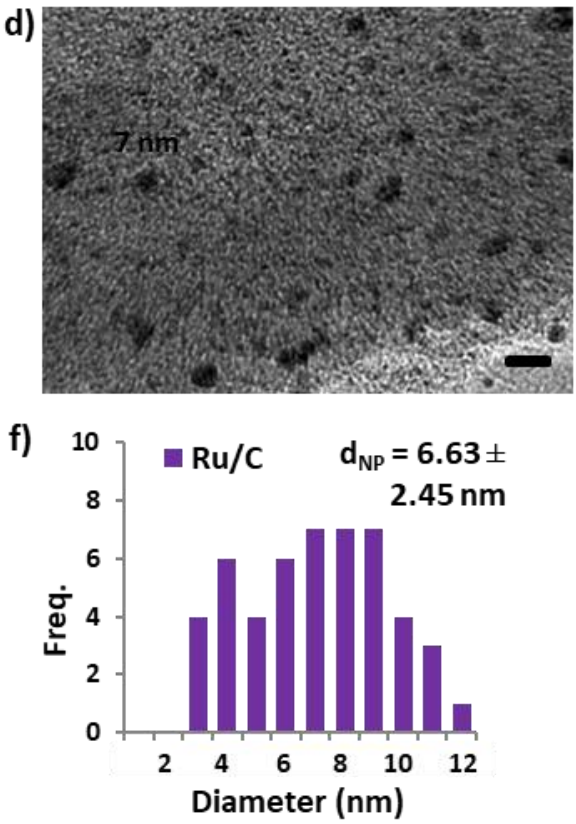

Figure 3. a-b) HRTEM images of RuNPs@GNF loaded with RuNPs (1 \% by wt), with enlarged region (b) showing the Ru [002] lattice spacing $(d=0.21 \mathrm{~nm})$ of an individual, hcp structured, metallic NP which corresponds to the $2 \theta$ peak centred at $42.2^{\circ}$ in the XRD (see ESI). Black arrows show RuNPs on the step edges inside the GNF. c) Dark-field scanning TEM image of RuNPs@GNF loaded with RuNPs. Only a very small amount of Ru material ( 7 $\%$ approximated by TEM analysis), is located on the outer surface of the GNF so all conversion is assumed to be a result of reactions catalysed by RuNPs on the inside of the GNF. d) HRTEM image of commercial Ru/C loaded with RuNPs (5 \% by wt.). White arrows show RuNPs located on the carbon support. e) The size distribution of RuNPs on the outside of the GNFs (green), and $\mathbf{d}$ ), the size distribution of RuNPs on the carbon (purple) shows the wide distribution of diameters of the RuNPs in the material. Scale bars: $10 \mathrm{~nm}$ in all images.

Previous studies have revealed that both the size and crystal structure of RuNPs have important effects on the catalytic activity of RuNPs in hydrogentation. ${ }^{42-45} \mathrm{Li}$ et al. reported that the size and loading of hcp RuNPs supported on mulit-walled carbon nanotubes for the hydrogentation of long-chain alkenes was optimum for 1.3 $\mathrm{nm}$ NPs and and metal loadings of $1 \%$ by wt., while in solution $3.1 \mathrm{~nm}$ RuNPs were observed to be the most active. ${ }^{42,43}$ Dupont et al. reported that $2.6 \pm 0.4 \mathrm{~nm}$ RuNPs were the most active for the partial hydrogenation of benzene, with TON of up to 165 for supported RuNPs catalysts. ${ }^{44}$ It is also observed that distortions to the lattice 
planes of hcp RuNPs ${ }^{45}$ or changes in crystal packing to face-centred cubic $\mathrm{Ru}^{36}$ results in significant effects to both the activity and selectivity of RuNP catalysed reactions.

Our hydronegation results, in which all RuNPs catalysts have the same hcp structure (confirmed by HRTEM, see ESI Figure S-5), show an interesting trend in activity, consistent for indivudal reactions with both alkene starting materials with TONs in the order of RuNPs@GNF>Ru/C>RuNPs@SWNT>RuNPs/C60@SWNT. It is important to highlight that the mass of catalyst in each reaction was scaled so the same molar percent of of Ru was present in all our reactions $(0.0017 \mathrm{mmol} \%$ of $\mathrm{Ru})$, and the trend in activity cannot be rationalised by simply considering the average NP size or total NP surface area for each sample, (for our series, the total surface area of RuNPs@SWNT>RuNPs/C60@SWNT>RuNPs@GNF>Ru/C), nor the extent of confinement, where the confinement in RuNPs@SWNT>RuNPs@GNF>RuNPs/C60@SWNT>Ru/C. Therefore the enhancement in activity observed upon confinement inside GNF must be a result of a balance of surface area of RuNP, confinemement of the reaction and accessibility of the catalytic centres. Indeed, GNF provides an optimum balance of RuNP confinement, leading to stabilisation and enhancement of local concentrations of rectants around the nanoparticles, and the ease of accessibility of the reactants to catalytic centres (hindered in the case of RuNP@SWNT). Recently, similar effects were observed in the oxygen reduction reaction catalysed by PtNP@GNF.46

In addition the RuNPs@GNFs catalyst shows a marked enhancement in reactivity for the production of $\mathbf{1 b}(\mathbf{1 b}: \mathbf{2 b}$ ratio $=2.7: 1)$ compared to the unconfined Ru/C catalyst (ratio $=2.4: 1)($ Table 2$)$ implying remarkable TON for $\mathbf{1 b}$, rationalised as a result of faster diffusion of the non-aromatic molecules to and from the step-edge compared to the aromatic benzonorbornadiene which may interact more strongly with the $\mathrm{sp}^{2}$ carbon of the step-edge and therefore diffuse to and from the RuNPs less rapidly. In contrast RuNPs@SWNT shows the opposite effect exhibiting and enhanced reactivity for the production of $\mathbf{2} \mathbf{b}(\mathbf{1} \mathbf{b}: \mathbf{2} \mathbf{b}$ ratio $=1.5: 1)$, potentially due to strong interactions between the aromatic core of $\mathbf{2 a}$ and the narrow inner channel of the SWNT.

In summary, these results reveal that the confinement imposed by the carbon nanoreactor can not only increase the activity of the Ru catalyst by both templating the formation of small, very active and stable RuNPs and potentially by increasing the local concentration of reactant molecules in the vicinity of the Ru catalyst but can also dramatically affect the efficiency of individual reactions depending on the size and shape of the reactant molecules. This is highlighted by the RuNPs@SWNT catalyst which shows that RuNPs confined in narrow SWNT are particularly efficient at converting aromatic $\mathbf{2 a}$ whilst the less constrained GNF catalyst are highly active and show enhanced relative conversion of 1a compared to the unconstrained (carbon black) reaction environment. Understanding the accessibility of the different catalysts is essential in order to fully appreciate the role that confinement plays in the performance of these materials.

These results are in agreement with previous observations that performing reactions in confinement has a number of important effects on catalysis and alters the outcome of reactions in a complex fashion; ${ }^{15}$ by enhancing the activity of nanaoparticle cataltysts, ${ }^{47}$ increasing the local concentration of reagents and thus increasing the rate of reactions, ${ }^{48}$ and by imposing restritions on both the transition states of intermediates ${ }^{41}$ and the flow of reactants in and products out of the nanoreactor. ${ }^{27}$ 
To investigate the 3D structure of the materials the experimentally measured active surface area of RuNPs@SWNT, RuNPs@GNF and commercial Ru/C catalysts were compared with theoretically calculated surface areas based on ideal models of the materials, i.e. assuming the entire catalyst is perfectly formed and accessible to reagents (see ESI for full details). Brunauer-Emmett-Teller (BET) measurements based on isothermal N2 gas adsorption at -196 ㄷ (77 K) were performed for RuNPs@SWNT, RuNPs@GNF and commercial $\mathrm{Ru} / \mathrm{C}$ catalysts along with control measurements of the support materials, i.e. SWNT, GNF and amorphous carbon materials to quantify both surface area (Table 3 ) and pore volume and size distribution (see supporting information Figure S-14-17 and Table 3-4). This provided a reasonable gauge of how much of the internal volume of the catalysts were accessible to gaseous $\mathrm{N}_{2}$, and comparison to the theoretical maximum values, revealed the portion of the internal volume which is either blocked by Ru nanoparticles large enough to completely fill the channels or other inherent material such as amorphous carbon and residual $\mathrm{Ni} / \mathrm{Y}$ catalyst present following the initial nanotube synthesis. BET measurements showed that the commercial $\mathrm{Ru} / \mathrm{C}\left(929 \mathrm{~m}^{2} / \mathrm{g}\right)$ has the largest surface compared to that of RuNPs@SWNT (352 m²/g) and RuNPs@GNF (29 m²/g) (see Table 3).

Table 3. Theoretical and BET surface area calculations for SWNT, GNF, RuNPs@SWNT, RuNPs@GNF and commercial $\mathrm{Ru} / \mathrm{C}$.

\begin{tabular}{lll}
\hline Catalyst & $\begin{array}{l}\text { Theoretical Surface Area } \\
\left(\mathbf{m}^{2} / \mathbf{g}\right)\end{array}$ & BET Surface Area $\left(\mathbf{m}^{2} / \mathbf{g}\right)$ \\
\hline SWNT $^{\mathrm{a}}$ & 2630.00 & 307.90 \\
RuNPs@SWNT & 2637.46 & 351.94 \\
C60@SWNT & 1315.00 & 120.99 \\
RuNPs/C60@SWNT & 1319.31 & 164.82 \\
GNF & & 12.08 \\
RuNPs@GNF & 35.00 & 28.53 \\
Carbon Black & 35.99 & 900.00 \\
Ru/C & N/A & 929.41 \\
\hline
\end{tabular}

a SWNT was annealed at $380{ }^{\circ} \mathrm{C}$ for 20 min prior to use. ${ }^{b} \mathrm{GNF}$ was annealed at $450{ }^{\circ} \mathrm{C}$ for 1 hour prior to use. ${ }^{\mathrm{C}}$ BET surface area value for the carbon black used to synthesize the $\mathrm{Ru} / \mathrm{C}$ was obtained from the manufacturer (Sigma-Aldrich).

The increased BET surface area of Ru/C and RuNPs@GNF compared to the background carbon and empty GNF supports respectively is attributed to the presence of the RuNPs. In contrast RuNPs@SWNT (352 m²/g) exhibited a lower BET surface area than that of the background SWNT $\left(508 \mathrm{~m}^{2} / \mathrm{g}\right)$. This is rationalised as a consequence of 
a small number of large (>1.2 nm) RuNPs blocking a significant percentage (c.a. 30-40 \%) of the entrances and/or channels/pores of the narrow nanotubes and not allowing $\mathrm{N}_{2}$ to access the full internal volume of the material.

Typically, carbon nanomaterials have very large surface areas due to their nanometre scale features; see theoretical specific surface calculated for each sample (Table S-3 in ESI). Peigney et. al. investigated the theoretical surface area of CNT and reported that the specific surface area of SWNT, independent of its diameter and length, is the same as that of both sides of a graphene sheet, i.e. $2630 \mathrm{~m}^{2} / \mathrm{g} .{ }^{49}$ As we opened the ends of the nanotubes prior to use, i.e. both internal and external walls of the nanotubes can be assumed to be accessible, the theoretical surface area for the SWNT sample is $2630 \mathrm{~m}^{2} / \mathrm{g}$. However, in reality the majority of SWNT form bundles and therefore it is difficult to approximate an accurate number for the theoretical surface area for nanotubes as the outer surface of most single nanotubes are not available as they are contained within the bundles. Clearly the experimental value for SWNT, $508 \mathrm{~m}^{2} / \mathrm{g}$, is dramatically lower and can be considered as the effect of bundling.

When considering the accessibility of GNFs it is important to understand that their structure is different to the concentric tubes of traditionally MWNTs and consists of stacked truncated cones of graphite layers arranged at an angle along the main axis. ${ }^{50}$ The internal surface has a succession of step edges which can act as anchoring points for guest molecules while the exterior surfaces are atomically flat. Therefore there is no reported framework for calculating a theoretical value for the surface area of GNFs.

In our approach we approximated the internal surfaces of a GNF to be flat and the sidewalls to consist of $\sim 75$ graphene layers (using an average thickness value of a GNF as $25 \mathrm{~nm}$ ). Therefore utilizing the same mathematical approach used by Peigney et al. for MWNTs, we calculated as theoretical surface area of $35 \mathrm{~m}^{2} / \mathrm{g}$, which is significantly higher that of the BET measurement $\left(12 \mathrm{~m}^{2} / \mathrm{g}\right)$. This is rationalized as the effect of the overlapping of the truncated cones in the internal channels decreasing the internal surface area compared to our theoretical model, but could also be a result of the presence of a number of GNFs in the sample with thicker sidewalls. Therefore, though it is difficult to comment on the RuNPs@SWNT sample, as a significant portion of the material appears to be blocked and inaccessible, c.f. an observed surface area of $>4$ times lower than predicted. This comparison does reveal that even though the $\mathrm{Ru} / \mathrm{C}$ catalyst has a higher surface area than RuNPs@GNF, both in terms of total catalyst and the RuNPs, it is significantly less active. This must be due to the size and shape of the pores, i.e. the nature of the confinement imposed.

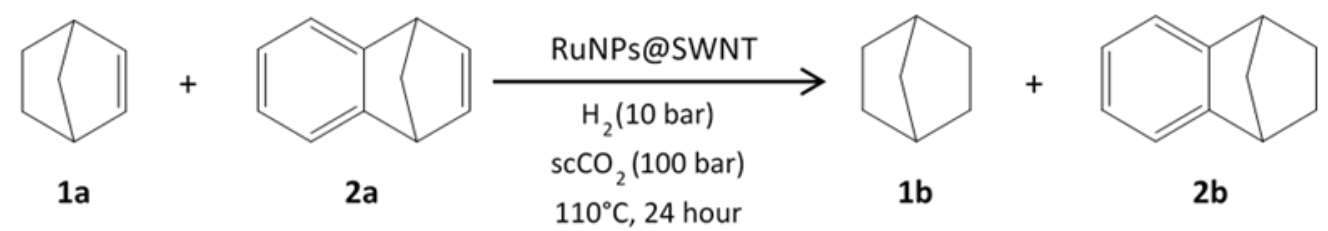

Scheme 2. Competitive reactions of norbornene and benzonorbornadiene in the presence RuNPs@SWNT using a $\mathrm{SCO}_{2}$ high pressure rig. 


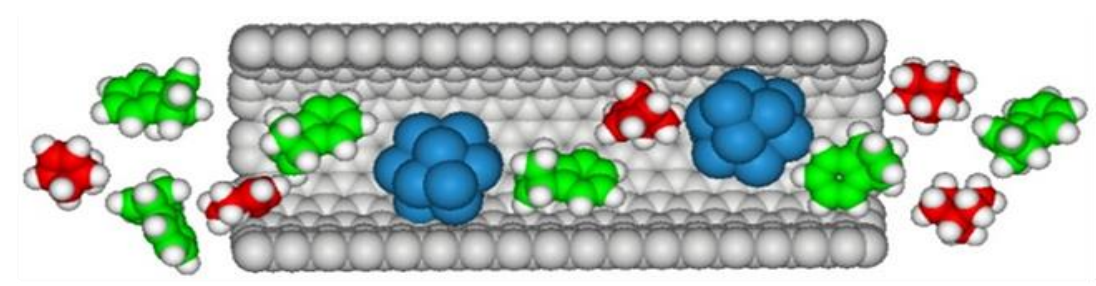

Figure 4. Schematic representation of the competitive hydrogenation of norbornene (red) and benzonorbornadiene (green) over RuNPs (blue) within SWNT. This shows how the extremely confined space within the nanotube channel traps the reactants close to the catalytic nanoparticles, effectively increasing the local concentration of reagents and increasing the rate of reaction.

Table 4. Competitive hydrogenation reactions of norbornene and benzonorbornadiene in the presence of RuNPs@SWNT, RuNPs/C60@SWNT, RuNPs@GNF and commercial Ru/C using a high pressure $\mathrm{scCO}_{2}$ batch reactor.

\begin{tabular}{|c|c|c|c|}
\hline \multirow[t]{2}{*}{ Catalyst } & \multicolumn{2}{|c|}{ Yield $^{\mathrm{a}}$ of Products (\%) / TON ${ }^{\mathrm{b}}$} & \multirow[t]{2}{*}{ Ratio of TONs for $\mathbf{1 b}: \mathbf{2 b}$} \\
\hline & $1 b$ & $2 b$ & \\
\hline RuNPs@SWNT & $70 / 1138$ & 89 / 1447 & $0.79: 1$ \\
\hline RuNPs/C60@SWNT & $7 / 197$ & 13 / 366 & $0.54: 1$ \\
\hline RuNPs@GNF & $21 / 2560$ & $42 / 5120$ & $0.5: 1$ \\
\hline $\mathrm{Ru} / \mathrm{C}$ & $52 / 3787$ & $53 / 3860$ & 0.98:1 \\
\hline
\end{tabular}

Reaction conditions; substrates ( $0.5 \mathrm{mmol}$ of each alkene), catalyst $\left(0.0017 \mathrm{~m}\right.$ mol\% of Ru in each reaction system), $\mathrm{H}_{2}(10$

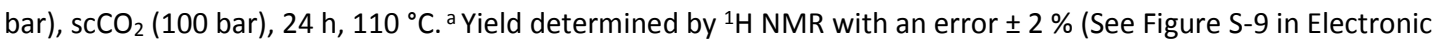

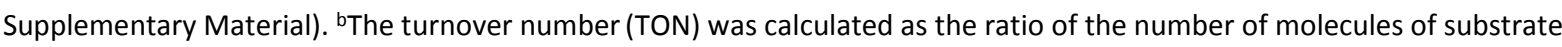
consumed in the reaction per theoretical number of true active Ru sites in catalyst used in the reaction.

To probe these effects of confinement further competitive reactions were investigated in which equimolar amounts of norbornene and benzonorbornadiene were simultaneously reduced within the nanoreactor catalyst (Scheme 2 and Figure 4).

Competitive reactions reveal that the confined space of carbon nanoreactors considerably affects the selectivity of the reactions compared to reactions performed in the absence of confinement (Ru/C) (Table 4). For all reactions in carbon nanoreactors benzonorbornadiene was preferentially reduced over norbornene in the presence of carbon nanoreactors due to the strong aromatic character of the nanotube. In fact the TONs of $\mathbf{2 b}$ in the competitive reactions are larger than the individual reactions for RuNPs@GNF and Ru/C despite a reduction in the starting concentration (Table 2), indicating that the presence of equimolar quatities of 1a may have an effect on $\pi-\pi$ interactions of the aromatic ring of $\mathbf{2 a}$ and the $\mathrm{sp}^{2}$ hybridized carbon network of the interior 
of the nanotubes being amplified in the rpesense of the non-aromatic 1 a leading to an increased local concentration of the aromatic reactant in proximity to RuNPs catalyst thus promoting reduction of $2 a^{41}$

RuNPs@GNF exhibited both the highest activity (total TON = 5680) and also the greatest selectivity towards $\mathbf{2 b}$ formation (ratio $\mathbf{1 b}: \mathbf{2} \mathbf{b}=0.5: 1$ ) compared to RuNPs@SWNT (ratio of 0.79:1) (Table 4). This can be explained by considering the nature of the confinement imposed by the step-edges of the internal channel of the GNF. Any increase in the local concentration of the reactants at the step-edges compared to the local concentration inside the nanotube cavity and bulk would result in a much higher rate of reaction. ${ }^{48}$ As shown in Figure $3 a$, the step-edges enable a very well-ordered distribution of RuNPs. The height of the step-edges, $\sim 3 \mathrm{~nm}, \mathrm{provides}$ a controlled space, which forms favourable $\pi-\pi$ interactions between the aromatic $\mathbf{2 a}$ and the sp $^{2}$ hybridized carbon step-edges and thus increases the concentration of $2 \mathbf{a}$ in the vicinity of the RuNPs and consequently increases the observed conversion of the aromatic compound. The same effect is observed for SWNT, with the formation of $\mathbf{2} \mathbf{b}$ observed to be enhanced compared to $\mathbf{1 b}$ in the individual reactions (see ratio of TONs in Table 2), however, the physical size of the aromatic compound means that there will be a significant steric barrier to the movement of the molecules into the narrow SWNT channels compared to the relatively larger space at the step edges in GNF which explains the lower observed TON for the SWNT catalyst.

\section{Conclusions}

In conclusion, a series of RuNPs@carbon nanoreactor catalysts have been synthesized and the catalytic properties of these materials have been assessed and the role of confinement explored. Overall, the confinement imposed by RuNPs@SWNT and RuNPs@GNF results in dramatic changes to reactions compared to commercially used $\mathrm{Ru} / \mathrm{C}$ which exhibits no confinement. The affinity of aromatic groups for the interior channels of carbon nanoreactors results in enhanced conversion of aromatic reagents in competitive hydrogenation reactions providing the ability to alter the selectivity of chemical reactions using support/reactant interactions.

Interestlingly the extreme confinement imposed by the shape of the SWNT nanoreactors is found to be a double edged sword, as though they exhibit enhanced selectivity towards aromatic substrates, as a result of strong interactions between the aromatic species and nanotube sidewall, the constricted space inside the SWNT lowers TONs in general. Remarkably it is the wider, structured interiors of GNF which have a greater effect on reactions, enhancing the activity and resulting in much higher TONs than the extremely narrow SWNT and thus offer the best of both worlds, in that they are wide enough not to limit diffusion of reactants but structured enough to impart the positive effects of reaction confinement.

\section{Acknowledgements}

This work was supported by Turkish Ministry of Education, European Research Council (ERC) and the EPSRC (EP/K005138/1 for the Kratos LiPPS XPS instrument). We would like to thank the Nanoscale and Microscale Research Centre (nmRC) for access to XPS, HRTEM and STEM instruments, Dr. A. La Torre for TEM support, Ben Pointer-Gleadhill for help with ICP-OES analysis, Dr. David Morgan for helpful discussions regarding the XPS peak assignments and Helena Matabosch Coromina for help to use the BET equipment. 
Electronic Supplementary Material: Supplementary material (containing additonal figures showing HRTEM images, size distribution and EDX data for RuNPs@SWNT, XPS data, XRD data for RuNPs@SWNT, RuNPs/C60@SWNT and RuNPs@GNF with the reference data obtained from the ICSD database of Royal Society of Chemistry, ${ }^{1} \mathrm{H}$ NMR spectra for competitive hydrogenation of norbornene and benzobnorbornadiene, TGA and ICP-OES data for RuNPs@SWNT, TGA data for RuNPs@GNF, details of the TON calculations used for single and competitive hydrogenation reactions, BET surface area, theoretical surface area calculations and pore size distributions for RuNPs@SWNT, RuNPs/C60@SWNT, RuNPs@GNF and Ru/C) is available in the online version of this article at ..

\section{References}

[1] D. K. Mishra, A. A. Dabbawala and J. S. Hwang, J. Mol. Catal. A: Chemical 2013, 376, 63-70.

[2] Q. Lin, G. Yang, Q. Chen, R. Fan, Y. Yoneyama, H. Wan and N. Tsubaki, Chem. Cat. Chem. 2015, 7, 682-689.

[3] I. Lee, M. A. Albiter, Q. Zhang, J. P. Ge, Y. D. Yin and F. Zaera, Phys. Chem. Chem. Phys. 2011, 13, 2449-2456.

[4] K. Okitsu, A. Yue, S. Tanabe and H. Matsumoto, Chem. Mater. 2000, 12, 3006-3011.

[5] M. Yoshizawa, J. K. Klosterman, and M. Fujita, Angew. Chem., Int. Ed. 2009, 48, 3418.

[6] N. A. Dhas, H. Cohen, and A. Gedanken, J. Phys. Chem. B 1997, 101, 6834-6838.

[7] B. S. Kong, J. X. Geng and H. T. Jung, Chem. Commun. 2009, 274-2176.

[8] G. M. Scheuermann, L. Rumi, P. Steurer, W. Bannwarth, and R. Mulhaupt, J. Am. Chem. Soc. 2009, 131, 8262-8270.

[9] X. Pan, and X. Bao, Acc. Chem. Res. 2011, 44, 553-562.

[10] D. A. Britz, A. N. Khlobystov, K. Porfyrakis, A. Ardavan and G. A. D. Briggs, Chem. Commun. 2005, 37-39.

[11] S. lijima, Nature 1991, 354.

[12] S. M. Dresselhaus, G. Dresselhaus and A. Jorio, Annu. Rev. Mater. Res. 2004, 34, 247-278.

[13] J. L. Figueiredo and M. F. R. Pereira, Carbon materials for catalysis, Carbon as Catalyst, in Carbon Materials for Catalysis (eds P. Serp and J. L. Figueiredo), John Wiley \& Sons, Inc., Hoboken, NJ, USA, 2008.

[14] J. Zhu, A. Holmen and D. Chen, Chem. Cat. Chem. 2013, 5, 378-401.

[15] A. N. Khlobystov, ACS Nano 2011, 5, 9306-9312.

[16] X. Pan, and X. Bao, Chem. Commun. 2008, 6271-6281.

[17] E. Castillejos, P. J. Debouttiere, L. Roiban, A. Solhy, V. Martinez, Y. Kihn, O. Ersen, K. Philippot, B. Chaudret and P. Serp, Angew. Chem., Int. Ed. 2009, 48, 2529-2533.

[18] W. A. Solomonsz, G. A. Rance, M. Suyetin, A. La Torre, E. Bichoutskaia and A. N. Khlobystov, Chem. Eur. J. 2012, 18, 13180-13187.

[19] B. Cornelio, A. R. Saunders, W. A. Solomonsz, M. Laronze-Cochard, A. Fontana, J. Sapi, A. N. Khlobystov and G. A. Rance, J. Mater. Chem. A. 2015, 3, 3918-3927. 
[20] D. Wang, G. Yang, O. Ma, M. Wu, Y. Tan, Y. Yoneyama and N. Tsubaki, ACS Catal. 2012, 2, 1958-1966.

[21] A. Benyounes, M. Kacimi, M. Ziyad and P. Serp, Chin. J. Catal. 2014, 35, 970-978.

[22] X. Pan, Z. Fan, W. Chen, Y. Ding, H. Luo and X. Bao, Nature Mater. 2007, 7, 507-511.

[23] J. Deng, P. Ren, D. Deng, L. Yu, F. Yang and X. Bao, Energy Environ. Sci. 2014, 7, 1919.

[24] T. W. Chamberlain, T. Zoberbier, J. Biskupek, A. Botos, U. Kaiser and A. N. Khlobystov, Chem. Sci. 2012, 3, 1919-1924.

[25] X. Li, T. Hungria C. G. Marcelot, M. R. Axet, P. -F. Fazzini, R. Tan, P. Serp, and K. Soulantica, Chem. Commun. 2016, 52, 2362-2365.

[26] I. Such-Basánez, M. C. Roman-Martínez and P. Serp, Microporous and Mesoporous Materials 2016, 225, 378-384.

[27] T. W. Chamberlain, J. H. Earley, D. P. Anderson, A. N. Khlobystov and R. A. Bourne, Chem. Commun. 2014, 50, 5200-5202.

[28] H. Zhang, X. Pan and X. Bao, Journal of Energy Chemistry 2013, 22, 251-256.

[29] H. Zhang, X. Pan, J. Liu, W. Qian, F. Wei, Y. Huang and X. Bao, ChemSusChem 2011, 4, 975-980.

[30] H. Zhang, X. Pan, X. Han. X. Liu, X. Wang, W. Shenand X. Bao, Chemical Science 2013, 4, 1075-1078.

[31] H. Shiozawa, T. Pichler, A. Grüneis, R. Pfeiffer, H. Kuzmany, Z. Liu, K. Suenaga and H. Kataura, Adv. Mater. 2008, 20, 1443-449.

[32] S. A. Miners, G. A. Rance and A. N. Khlobystov, Chem. Commun. 2013, 49, 5586-5588.

[33] S. A. Miners, G. A. Rance, A. La Torre, S. M. Kenny, A. N. Khlobystov, J. Mater. Chem. C, 2014, 2, 8357-8363.

[34] J. W. Coe, M. C. Wirtz, C. G. Bashore and J. Candler, Org. Lett. 2004, 6(10), 1589-92.

[35] E. Castillejos, P. J. Debouttiere, L. Roiban, A. Solhy, V. Martinez, Y. Kihn, O. Ersen, K. Philippot, B. Chaudret, P. Serp, Angew. Chem., Int. Ed. 2009, 48, 2529-2533.

[36] K. Kusada, H. Kobayashi, T. Yamamoto, S. Matsumura, N. Sumi, K. Sato, K. Nagaoka, Y. Kubota, H. Kitagawa, J. Am. Chem. Soc. 2013, 135, 5493-5496.

[37] W. Chen and S. W. Chen, Angew. Chem. Int., Ed. 2009, 48, 4386-4389.

[38] R. S. Oosthuizen and V. O. Nyamori, Platinum Metals Rev. 2011, 55, 154-169.

[39] Z. -Q. Feng, X. -L. Yang, Y. -F. Ye and L. -Y. Hao, Bull. Korean Chem. Soc. 2014, 35, No. 4.

[40] J. F. Moulder, W. F. Stickle, P. E. Sobol and K. D. Bomben, 1992 Handbook of X-ray Photoelectron Spectroscopy (Perkin-Elmer, Minnesota) pp. 44 and 114.

[41] M. A. Lebedeva, T. W. Chamberlain. A. Thomas B. E. Thomas, C. T. Stoppiello, E. Volkova, M. Suyetin, A. N. Khlobystov, Nanoscale 2016, 8, 11727-11737.

[42] S. Guo, K. Y. Liew, J. Li, J Am Oil Chem Soc 2009, 86, 1141-1147.

[43] B. Xu, K. Y. Liew, J. Li, J Am Oil Chem Soc 2007, 84, 117-122. 
[44] E. T.Silveira, A. P.Umpierre, L. M. Rossi, G. Machado, J. Morais, G. V. Soares, I. J. R. Baumvol, S. R. Teixeira, P. F.P. Fichtner, J. Dupont, Chem. Eur. J. 2004, 10, 3734-3740.

[45] J. Mao, W. Chen, W. Sun, Z. Chen, J. Pei, D. He, C. Lv, D. Wang, Y. Li Angew. Chem., Int. Ed., 2017, 56, 1-6.

[46] M. C. Gimenez-Lopez, A. Kurtoglu, D. A. Walsh, A. N. Khlobystov Adv. Mat. 2016, 28, 9103-9108.

[47] S. Guo, X. Pan, H. Gao, Z. Yang, J. Zhao, X. Bao, Chem Eur J 2010, 6, 5379-5384.

[48] M. J. Ledoux, R. Vieira, C. Pham-Huu, N. Keller, J. Catal. 2003, 216, 333-342.

[49] A. Peigney, C. Laurent, E. Flahaut, R. Bacsa and A. Rousset, Carbon, Elsevier 2001, 39, 507-514.

[50] A. La Torre, M. C. Gimenez-Lopez, M. W. Fay, G. A. Rance, W. A. Solomonsz, T. W. Chamberlain, P. D. Brown and A. N. Khlobystov, ACS Nano 2012, 6, 2000-2007. 